\title{
Soins péri-opératoires en chirurgie colorectale Conclusion
}

\section{J. H. Lefevre}

(C) Lavoisier SAS 2015

Voilà, vous savez tout sur les soins péri-opératoires.

N'oubliez pas, c'est un ensemble de mesures à intégrer dans vos pratiques.

Prendre une ou deux idées ne seront pas efficaces et risquent même de vous freiner dans votre envie d'améliorer votre prise en charge.

La plupart sont, comme vous avez pu le lire, assez simples et facilement intégrables. Vous pouvez débuter par le dépistage de la carence martiale chez vos patients $(50 \%)$, rechercher une perte de poids. Une perfusion de fer et une renutri- tion pendant une dizaine de jours peuvent déjà réduire votre taux de transfusions postopératoires et votre morbidité.

Pour les modalités du programme ERAS, tout est détaillé dans les deux chapitres de ce dossier et également disponibles sur le site de l'association.

N'hésitez pas enfin à évaluer vos pratiques ... Quels sont vos taux de tranfusions après colectomies et votre durée moyenne de séjour?

Et pourquoi ne pas faire sortir votre prochaine colectomie sous cœlioscopie au $3^{\mathrm{e}}$ jour? 